\title{
Derived Equivalences of Triangular Matrix Rings Arising from Extensions of Tilting Modules
}

\author{
Sefi Ladkani
}

Received: 1 September 2008 / Accepted: 19 December 2008 / Published online: 16 December 2009

(C) The Author(s) 2009. This article is published with open access at Springerlink.com

\begin{abstract}
A triangular matrix ring $\Lambda$ is defined by a triplet $(R, S, M)$ where $R$ and $S$ are rings and ${ }_{R} M_{S}$ is an $S$ - $R$-bimodule. In the main theorem of this paper we show that if $T_{S}$ is a tilting $S$-module, then under certain homological conditions on the $S$-module $M_{S}$, one can extend $T_{S}$ to a tilting complex over $\Lambda$ inducing a derived equivalence between $\Lambda$ and another triangular matrix ring specified by $\left(S^{\prime}, R, M^{\prime}\right)$, where the ring $S^{\prime}$ and the $R$ - $S^{\prime}$-bimodule $M^{\prime}$ depend only on $M$ and $T_{S}$, and $S^{\prime}$ is derived equivalent to $S$. Note that no conditions on the ring $R$ are needed. These conditions are satisfied when $S$ is an Artin algebra of finite global dimension and $M_{S}$ is finitely generated. In this case, $\left(S^{\prime}, R, M^{\prime}\right)=(S, R, D M)$ where $D$ is the duality on the category of finitely generated $S$-modules. They are also satisfied when $S$ is arbitrary, $M_{S}$ has a finite projective resolution and $\operatorname{Ext}_{S}^{n}\left(M_{S}, S\right)=0$ for all $n>0$. In this case, $\left(S^{\prime}, R, M^{\prime}\right)=\left(S, R, \operatorname{Hom}_{S}(M, S)\right)$.
\end{abstract}

Keywords Triangular matrix ring $\cdot$ Derived equivalence $\cdot$ Tilting complex

Mathematics Subject Classifications (2000) $18 \mathrm{E} 30 \cdot 16 \mathrm{~S} 50 \cdot 18 \mathrm{~A} 25$

\section{Introduction}

Triangular matrix rings and their homological properties have been widely studied, see for example $[4,7,8,16,18]$. The question of derived equivalences between differ-

Presented by Claus Ringel.

S. Ladkani $(\bowtie)$

Einstein Institute of Mathematics, The Hebrew University of Jerusalem, Jerusalem 91904, Israel e-mail: sefil@math.huji.ac.il

Present Address:

S. Ladkani

Max-Planck-Institut für Mathematik, Vivatsgasse 7, 53111 Bonn, Germany

e-mail: sefil@mpim-bonn.mpg.de 
ent such rings was explored in the special case of one-point extensions of algebras [2]. Another aspect of this question was addressed by considering examples of triangular matrix algebras of a simple form, such as incidence algebras of posets [14]. In this paper we extend the results of [14] to general triangular matrix rings.

A triangular matrix ring $\Lambda$ is defined by a triplet $(R, S, M)$ where $R$ and $S$ are rings and ${ }_{R} M_{S}$ is an $S$ - $R$-bimodule. The category of (right) $\Lambda$-modules can be viewed as a certain gluing of the categories of $R$-modules and $S$-modules, specified by four exact functors. This gluing naturally extends to the bounded derived categories. We note the similarity to the classical "recollement" situation, introduced by Beilinson, Bernstein and Deligne [3], involving six functors between three triangulated categories, originally inspired by considering derived categories of sheaves on topological spaces, and later studied for derived categories of modules by Cline, Parshall and Scott $[5,6]$, see also [13].

In Section 2 we show that triangular matrix rings arise naturally as endomorphism rings of certain rigid complexes over abelian categories that are glued from two simpler ones. Here, a complex $T \in \mathscr{D}^{b}(\mathscr{C})$ is rigid if $\operatorname{Hom}_{\mathscr{D}^{b}(\mathscr{C})}(T, T[n])=0$ for all $n \neq 0$, where $\mathscr{D}^{b}(\mathscr{C})$ denotes the bounded derived category of an abelian category $\mathscr{C}$. Similarly, an object $T \in \mathscr{C}$ is rigid if it is rigid as a complex.

Indeed, when $\mathscr{C}$ is glued from the abelian categories $\mathscr{A}$ and $\mathscr{B}$, we construct, for any projective object of $\mathscr{A}$ and a rigid object of $\mathscr{B}$ satisfying some homological conditions, a new rigid complex in $\mathscr{D}^{b}(\mathscr{C})$ whose endomorphism ring is a triangular matrix ring.

In particular, as demonstrated in Section 3, this construction applies for comma categories defined by two abelian categories $\mathscr{A}, \mathscr{B}$ and a right exact functor $F$ : $\mathscr{A} \rightarrow \mathscr{B}$. In this case, any projective $P$ of $\mathscr{A}$ and a rigid object $T_{\mathscr{B}} \in \mathscr{B}$ satisfying $\operatorname{Ext}_{\mathscr{B}}^{n}\left(F P, T_{\mathscr{B}}\right)=0$ for all $n>0$, give rise to a rigid complex $T$ over the comma category, whose endomorphism ring is a triangular matrix ring which can be explicitly computed in terms of $P, T_{\mathscr{B}}$ and $F P$, as

$$
\operatorname{End}_{\mathscr{D}^{b}(\mathscr{C})}(T) \simeq\left(\begin{array}{cc}
\operatorname{End}_{\mathscr{B}}\left(T_{\mathscr{B}}\right) & \operatorname{Hom}_{\mathscr{B}}\left(F P, T_{\mathscr{B}}\right) \\
0 & \operatorname{End}_{\mathscr{A}}(P)
\end{array}\right)
$$

In Section 4 we apply this construction for categories of modules over triangular matrix rings. For a ring $\Lambda$, denote by $\operatorname{Mod} \Lambda$ the category of all right $\Lambda$-modules, and by $\mathscr{D}^{b}(\Lambda)$ its bounded derived category. Recall that a complex $T \in \mathscr{D}^{b}(\Lambda)$ is a tilting complex if it is rigid and moreover, the smallest full triangulated subcategory of $\mathscr{D}^{b}(\Lambda)$ containing $T$ and closed under forming direct summands, equals per $\Lambda$, the full subcategory in $\mathscr{D}^{b}(\Lambda)$ of complexes quasi-isomorphic to perfect complexes, that is, bounded complexes of finitely generated projective $\Lambda$-modules. If, in addition, $H^{n}(T)=0$ for all $n \neq 0$, we call $T$ a tilting module and identify it with the module $H^{0}(T)$.

Two rings $\Lambda$ and $\Lambda^{\prime}$ are derived equivalent if $\mathscr{D}^{b}(\Lambda)$ and $\mathscr{D}^{b}\left(\Lambda^{\prime}\right)$ are equivalent as triangulated categories. By Rickard's Morita theory for derived equivalence [19], this is equivalent to the existence of a tilting complex $T \in \mathscr{D}^{b}(\Lambda)$ such that $\operatorname{End}_{\mathscr{D}^{b}(\Lambda)}(T)=\Lambda^{\prime}$.

When $\Lambda$ is a triangular matrix ring defined by two rings $R, S$ and a bimodule ${ }_{R} M_{S}$, the category $\operatorname{Mod} \Lambda$ is the comma category of $\operatorname{Mod} R, \operatorname{Mod} S$ with respect to the functor $-\otimes M: \operatorname{Mod} R \rightarrow \operatorname{Mod} S$. In this case, starting with the projective $R$-module $R$ and a tilting $S$-module $T_{S}$, the complex $T$ constructed in Section 3 is not only rigid, 
but also a tilting complex, hence we deduce a derived equivalence between $\Lambda$ and the triangular matrix ring End $_{\mathscr{D}^{b}(\Lambda)}(T)$, as expressed in the theorem below, repeated as Theorem 4.5 in the body of the paper.

Theorem Let $R, S$ be rings and $T_{S}$ a tilting $S$-module. Let ${ }_{R} M_{S}$ be an $S$ - $R$-bimodule such that as an $S$-module, $M_{S} \in \operatorname{per} S$ and $\operatorname{Ext}_{S}^{n}\left(M_{S}, T_{S}\right)=0$ for all $n>0$. Then the triangular matrix rings

$$
\Lambda=\left(\begin{array}{cc}
R & M \\
0 & S
\end{array}\right) \quad \text { and } \quad \widetilde{\Lambda}=\left(\begin{array}{cc}
\operatorname{End}_{S}\left(T_{S}\right) & \operatorname{Hom}_{S}\left(M, T_{S}\right) \\
0 & R
\end{array}\right)
$$

are derived equivalent.

We note that the assumption that $T_{S}$ is a tilting module implies that the rings $S$ and $\operatorname{End}_{S}\left(T_{S}\right)$ are derived equivalent, hence the triangular matrix ring specified by the triplet $(R, S, M)$ is derived equivalent to one specified by $\left(S^{\prime}, R, M^{\prime}\right)$ where $S^{\prime}$ is derived equivalent to $S$. We note also that no conditions on the $\operatorname{ring} R$ (or on $M$ as a left $R$-module) are necessary.

The above theorem has two interesting corollaries, corresponding to the cases where $T_{S}$ is injective or projective.

For the first corollary, let $S$ be an Artin algebra, and let $D: \bmod S \rightarrow \bmod S^{o p}$ denote the duality. When $S$ has finite global dimension, one can take $T_{S}$ to be the module $D S$ which is then an injective tilting module.

Corollary Let $R$ be a ring, $S$ an Artin algebra with $\operatorname{gl} \operatorname{dim} S<\infty$ and ${ }_{R} M_{S}$ an $S$ - $R$ bimodule which is finitely generated as an $S$-module. Then the triangular matrix rings

$$
\Lambda=\left(\begin{array}{cc}
R & M \\
0 & S
\end{array}\right) \quad \text { and } \quad \widetilde{\Lambda}=\left(\begin{array}{cc}
S & D M \\
0 & R
\end{array}\right)
$$

are derived equivalent, where $D$ is the duality on $\bmod S$.

This corollary is repeated as Theorem 4.9. The ring $\widetilde{\Lambda}$ depends only on $R, S$ and $M$, hence it may be considered as a derived equivalent mate of $\Lambda$.

The second corollary of the above theorem is obtained by taking the tilting $S$ module to be $S$.

Corollary Let $R, S$ be rings and ${ }_{R} M_{S}$ an $S$ - $R$-bimodule such that as an $S$-module, $M_{S} \in \operatorname{per} S$ and $\operatorname{Ext}_{S}^{n}\left(M_{S}, S\right)=0$ for all $n>0$. Then the triangular matrix rings

$$
\Lambda=\left(\begin{array}{cc}
R & M \\
0 & S
\end{array}\right) \quad \text { and } \quad \tilde{\Lambda}=\left(\begin{array}{cc}
S & \operatorname{Hom}_{S}(M, S) \\
0 & R
\end{array}\right)
$$

are derived equivalent.

This corollary, repeated as Corollary 4.11, applies to the following situations, listed in descending order of generality; the ring $S$ is self-injective (that is, $S$ is injective as a module over itself) and $M_{S}$ is finitely generated projective; the ring $S$ is semi-simple and $M_{S}$ is finitely generated; the ring $S$ is a division ring and $M$ is finite dimensional 
over $S$. The latter case implies that a triangular matrix ring which is a one-point extension is derived equivalent to the corresponding one-point co-extension.

In Section 5 we conclude with three remarks concerning the specific case of finite dimensional triangular matrix algebras over a field.

First, in the case when $R$ and $S$ are finite dimensional algebras over a field and both have finite global dimension, an alternative approach to show the derived equivalence of the triangular matrix algebras specified by $(R, S, M)$ and its mate $(S, R, D M)$ is to prove that the corresponding repetitive algebras are isomorphic and then use Happel's Theorem [9, (II,4.9)]. However, in the case that only one of $R$ and $S$ has finite global dimension, Happel's Theorem cannot be used, but the derived equivalence still holds. Moreover, as we show in Example 5.3, there are cases when none of $R$ and $S$ have finite global dimension and the corresponding algebras are not derived equivalent, despite the isomorphism between their repetitive algebras.

Second, one can directly prove, using only matrix calculations, that when at least one of $R$ and $S$ has finite global dimension, the Cartan matrices of the triangular matrix algebra $(R, S, M)$ and its mate are equivalent over $\mathbb{Z}$, a result which is a direct consequence of Theorem 4.9 .

Third, we note that in contrast to triangular matrix algebras, in the more general situation of trivial extensions of algebras, the mates $A \ltimes M$ and $A \ltimes D M$ for an algebra $A$ and a bimodule ${ }_{A} M_{A}$, are typically not derived equivalent.

\section{The Gluing Construction}

Let $\mathscr{A}, \mathscr{B}, \mathscr{C}$ be three abelian categories. Similarly to [3, (1.4)], we view $\mathscr{C}$ as glued from $\mathscr{A}$ and $\mathscr{B}$ if there exist certain functors $i^{-1}, i_{*}, j^{-1}, j_{\text {! }}$ as described below. Note, however, that we start by working at the level of the abelian categories and not their derived categories. In addition, the requirement in [3] of the existence of the additional adjoint functors $i^{!}, j_{*}$ is replaced by the orthogonality condition (2.6).

Definition 2.1 A quadruple of additive functors $i^{-1}, i_{*}, j^{-1}, j$ ! as in the diagram

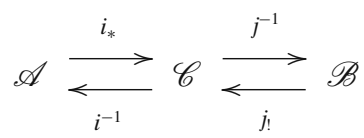

is called gluing data if it satisfies the four properties (2.1.1)-(2.1.4) below.

\subsubsection{Adjunction}

$i^{-1}$ is a left adjoint of $i_{*}$ and $j^{-1}$ is a right adjoint of $j$ ! That is, there exist bi-functorial isomorphisms

$$
\begin{aligned}
& \operatorname{Hom}_{\mathscr{A}}\left(i^{-1} C, A\right) \simeq \operatorname{Hom}_{\mathscr{C}}\left(C, i_{*} A\right) \\
& \operatorname{Hom}_{\mathscr{B}}\left(B, j^{-1} C\right) \simeq \operatorname{Hom}_{\mathscr{C}}(j ! B, C)
\end{aligned}
$$

for all $A \in$ ob $\mathscr{A}, B \in$ ob $\mathscr{B}, C \in$ ob $\mathscr{C}$. 


\subsubsection{Exactness}

The functors $i^{-1}, i_{*}, j^{-1}, j$ ! are exact.

Note that by the adjunctions above, we automatically get that the functors $i_{*}, j^{-1}$ are left exact while $i^{-1}, j$ ! are right exact.

\subsubsection{Extension}

For every $C \in$ ob $\mathscr{C}$, the adjunction morphisms $j_{!} j^{-1} C \rightarrow C$ and $C \rightarrow i_{*} i^{-1} C$ give rise to a short exact sequence

$$
0 \rightarrow j_{!} j^{-1} C \rightarrow C \rightarrow i_{*} i^{-1} C \rightarrow 0
$$

\subsubsection{Orthogonality}

$$
\begin{array}{ll}
i^{-1} j_{!}=0 & j^{-1} i_{*}=0 \\
i^{-1} i_{*} \simeq \operatorname{Id}_{\mathscr{A}} & j^{-1} j_{!} \simeq \operatorname{Id}_{\mathscr{B}}
\end{array}
$$

and in addition,

$$
\operatorname{Hom}_{\mathscr{C}}\left(i_{*} A, j_{!} B\right)=0 \quad \text { for all } A \in \mathrm{ob} \mathscr{A}, B \in \mathrm{ob} \mathscr{B}
$$

Using the adjunctions (2.1) and (2.2), the assumptions of Eqs. 2.4, 2.5 can be rephrased as follows. First, the two conditions of Eq. 2.4 are equivalent to each other and each is equivalent to the condition

$$
\operatorname{Hom}_{\mathscr{C}}\left(j ! B, i_{*} A\right)=0 \quad \text { for all } A \in \mathrm{ob} \mathscr{A}, B \in \mathrm{ob} \mathscr{B}
$$

Similarly, the conditions in Eq. 2.5 are equivalent to the requirement that $i_{*}$ and $j$ ! are fully faithful functors, so that one can think of $\mathscr{A}$ and $\mathscr{B}$ as embedded in $\mathscr{C}$. Moreover, from Eqs. 2.3 and 2.7 we see that $(\mathscr{B}, \mathscr{A})$ is a torsion pair [10, (I.2)] in $\mathscr{C}$.

Observe also that Eq. 2.6 could be replaced with the assumption that the functor $\left(i^{-1}, j^{-1}\right): \mathscr{C} \rightarrow \mathscr{A} \times \mathscr{B}$ is faithful. Indeed, one implication follows from Eqs. 2.4 and 2.5, and the other follows using Eq. 2.3.

From now on assume that $\left(i^{-1}, i_{*}, j^{-1}, j_{!}\right)$form a gluing data.

Lemma 2.2 If $P$ is a projective object of $\mathscr{C}$, then $i^{-1} P$ is projective in $\mathscr{A}$. Similarly, if $I$ an injective object of $\mathscr{C}$, then $j^{-1} I$ is injective in $\mathscr{B}$.

Proof A functor which is a left adjoint to an exact functor preserves projectives, while a right adjoint to an exact functor preserves injectives [8, Corollary 1.6].

The exact functors $i^{-1}, i_{*}, j^{-1}, j$ ! give rise to triangulated functors between the corresponding bounded derived categories. We use the same notation for these derived functors:

$$
\mathscr{D}^{b}(\mathscr{A}) \underset{i^{-1}}{\stackrel{i_{*}}{\rightleftarrows}} \mathscr{D}^{b}(\mathscr{C}) \underset{j !}{\stackrel{j^{-1}}{\longleftarrow}} \mathscr{D}^{b}(\mathscr{B})
$$


Note that adjunctions and orthogonality relations analogous to Eqs. 2.1, 2.2, 2.4, 2.5 (but not Eq. 2.6) hold also for the derived functors. In particular, $i_{*}$ and $j !$ are fully faithful.

Definition 2.3 An object $T$ in an abelian category $\mathscr{A}$ is called rigid if $\operatorname{Ext}_{\mathscr{A}}^{n}(T, T)=0$ for all $n>0$.

Proposition 2.4 Let $P$ be a projective object of $\mathscr{C}$ and $T_{\mathscr{B}}$ be a rigid object of $\mathscr{B}$ such that $\operatorname{Ext}_{\mathscr{B}}^{n}\left(j^{-1} P, T_{\mathscr{B}}\right)=0$ for all $n>0$. Consider the complex

$$
T=i_{*} i^{-1} P \oplus j ! T_{\mathscr{B}}[1]
$$

in $\mathscr{D}^{b}(\mathscr{C})$. Then $\operatorname{Hom}_{\mathscr{D}^{b}(\mathscr{C})}(T, T[n])=0$ for $n \neq 0$ and

$$
\operatorname{End}_{\mathscr{D}^{b}(\mathscr{C})}(T) \simeq\left(\begin{array}{cc}
\operatorname{End}_{\mathscr{B}}\left(T_{\mathscr{B}}\right) & \operatorname{Ext}_{\mathscr{C}}^{1}\left(i_{*} i^{-1} P, j !\right. \\
0 & \operatorname{End}_{\mathscr{A}}\left(i^{-1} P\right)
\end{array}\right)
$$

is a triangular matrix ring.

Proof Since $T$ has two summands, the space $\operatorname{Hom}_{\mathscr{D}^{b}(\mathscr{C})}(T, T[n])$ decomposes into four parts, which we now consider.

Since $i_{*}$ is fully faithful and $i^{-1} P$ is projective,

$$
\operatorname{Hom}_{\mathscr{D}^{b}(\mathscr{C})}\left(i_{*} i^{-1} P, i_{*} i^{-1} P[n]\right) \simeq \operatorname{Hom}_{\mathscr{D}^{b}(\mathscr{A})}\left(i^{-1} P, i^{-1} P[n]\right)
$$

vanishes for $n \neq 0$. Similarly, since $j !$ is fully faithful and $T_{\mathscr{B}}$ is rigid,

$$
\operatorname{Hom}_{\mathscr{D}^{b}(\mathscr{C})}\left(j ! T_{\mathscr{B}}, j ! T_{\mathscr{B}}[n]\right) \simeq \operatorname{Hom}_{\mathscr{D}^{b}(\mathscr{B})}\left(T_{\mathscr{B}}, T_{\mathscr{B}}[n]\right)
$$

vanishes for $n \neq 0$. Moreover, by orthogonality,

$$
\operatorname{Hom}_{\mathscr{D}^{b}(\mathscr{C})}\left(j_{!} T_{\mathscr{B}}, i_{*} i^{-1} P[n]\right)=0
$$

for all $n \in \mathbb{Z}$.

It remains to consider $\operatorname{Hom}_{\mathscr{D}^{b}(\mathscr{C})}\left(i_{*} i^{-1} P, j_{!} T_{\mathscr{B}}[n]\right)$ and to prove that it vanishes for $n \neq 1$. Using Eq. 2.3, we obtain a short exact sequence $0 \rightarrow j ! j^{-1} P \rightarrow P \rightarrow i_{*} i^{-1} P \rightarrow$ 0 that induces a long exact sequence, a fragment of which is shown below:

$$
\begin{array}{r}
\operatorname{Hom}_{\mathscr{D}^{b}(\mathscr{B})}\left(j^{-1} P, T_{\mathscr{B}}[n-1]\right) \simeq \operatorname{Hom}_{\mathscr{D}^{b}(\mathscr{C})}\left(j_{!} j^{-1} P[1], j_{!} T_{\mathscr{B}}[n]\right) \\
\rightarrow \operatorname{Hom}_{\mathscr{D}^{b}(\mathscr{C})}\left(i_{*} i^{-1} P, j ! T_{\mathscr{B}}[n]\right) \rightarrow \operatorname{Hom}_{\mathscr{D}^{b}(\mathscr{C})}\left(P, j_{!} T_{\mathscr{B}}[n]\right) .
\end{array}
$$

Now observe that the right term vanishes for $n \neq 0$ since $P$ is projective, and the left term of Eq. 2.9 vanishes for $n \neq 1$ by our assumption on the vanishing of $\operatorname{Ext}_{\mathscr{B}}^{\bullet}\left(j^{-1} P, T_{\mathscr{B}}\right)$. Therefore

$$
\operatorname{Hom}_{\mathscr{D}^{b}(\mathscr{C})}\left(i_{*} i^{-1} P, j ! T_{\mathscr{B}}[n]\right)=0
$$

for $n \neq 0,1$. This holds also for $n=0$ by the assumption (2.6).

To complete the proof, note that $\operatorname{Ext}_{\mathscr{C}}^{1}\left(i_{*} i^{-1} P, j_{!} T_{\mathscr{B}}\right)$ has a natural structure of an $\operatorname{End}_{\mathscr{A}}\left(i^{-1} P\right)$-End ${ }_{\mathscr{B}}\left(T_{\mathscr{B}}\right)$-bimodule via the identifications

$$
\operatorname{End}_{\mathscr{A}}\left(i^{-1} P\right) \simeq \operatorname{End}_{\mathscr{C}}\left(i_{*} i^{-1} P\right) \quad \operatorname{End}_{\mathscr{B}}\left(T_{\mathscr{B}}\right) \simeq \operatorname{End}_{\mathscr{C}}\left(j_{!} T_{\mathscr{B}}\right)
$$


Remark 2.5 The assumptions in the proposition are always satisfied when $P$ is a projective object of $\mathscr{C}$ and $T_{\mathscr{B}}$ is any injective object of $\mathscr{B}$.

Remark 2.6 One can formulate an analogous statement for a rigid object $T_{\mathscr{A}}$ of $\mathscr{A}$ and an injective object $I$ of $\mathscr{C}$.

\section{Gluing in Comma Categories}

Let $\mathscr{A}, \mathscr{B}$ be categories and $F: \mathscr{A} \rightarrow \mathscr{B}$ a functor. The comma category with respect to the pair of functors $\mathscr{A} \stackrel{F}{\rightarrow} \mathscr{B} \stackrel{\text { Id }}{\leftarrow} \mathscr{B}[15$, II.6], denoted by ( $F \downarrow$ Id), is the category $\mathscr{C}$ whose objects are triples $(A, B, f)$ where $A \in$ ob $\mathscr{A}, B \in$ ob $\mathscr{B}$ and $f: F A \rightarrow B$ is a morphism (in $\mathscr{B})$. The morphisms between objects $(A, B, f)$ and $\left(A^{\prime}, B^{\prime}, f^{\prime}\right)$ are all pairs of morphisms $\alpha: A \rightarrow A^{\prime}, \beta: B \rightarrow B^{\prime}$ such that the square

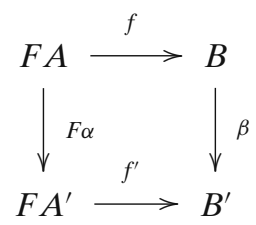

commutes.

Assume in addition that $\mathscr{A}, \mathscr{B}$ are abelian and that $F: \mathscr{A} \rightarrow \mathscr{B}$ is an additive, right exact functor. In this case, the comma category $\mathscr{C}$ is abelian [8]. Consider the functors

$$
\mathscr{A} \underset{i^{-1}}{\stackrel{i_{*}}{\longrightarrow}} \mathscr{C} \underset{j !}{\stackrel{j^{-1}}{\gtrless}} \mathscr{B}
$$

defined by

$$
\begin{array}{cccc}
i_{*}(A)=(A, 0,0) & i_{*}(\alpha)=(\alpha, 0) & i^{-1}(A, B, f)=A & i^{-1}(\alpha, \beta)=\alpha \\
j !(B)=(0, B, 0) & j !(\beta)=(0, \beta) & j^{-1}(A, B, f)=B & j^{-1}(\alpha, \beta)=\beta
\end{array}
$$

for objects $A \in$ ob $\mathscr{A}, B \in$ ob $\mathscr{B}$ and morphisms $\alpha, \beta$.

Lemma 3.1 The quadruple $\left(i^{-1}, i_{*}, j^{-1}, j_{!}\right)$is a gluing data.

Proof We need to verify the four properties of gluing data. The adjunction follows by the commutativity of the diagrams
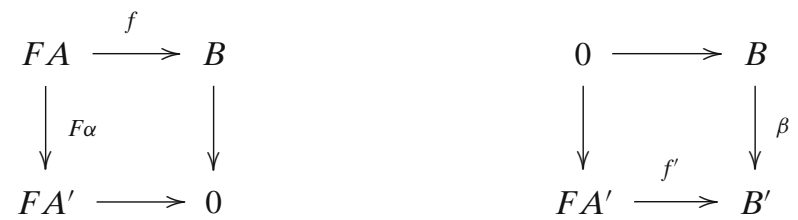

for $\alpha: A \rightarrow A^{\prime}$ and $\beta: B \rightarrow B^{\prime}$. 
For exactness, note that kernels and images in $\mathscr{C}$ can be computed componentwise, that is, if $(\alpha, \beta):(A, B, f) \rightarrow\left(A^{\prime}, B^{\prime}, f^{\prime}\right)$ is a morphism in $\mathscr{C}$, then $\operatorname{ker}(\alpha, \beta)=$ $\left(\operatorname{ker} \alpha, \operatorname{ker} \beta,\left.f\right|_{F(\operatorname{ker} \alpha)}\right)$ and similarly for the image. The extension condition follows from

$$
0 \rightarrow(0, B, 0) \stackrel{\left(0,1_{B}\right)}{\longrightarrow}(A, B, f) \stackrel{\left(1_{A}, 0\right)}{\longrightarrow}(A, 0,0) \rightarrow 0
$$

and orthogonality is straightforward.

One can use the special structure of the comma category $\mathscr{C}$ to define another pair of functors. Let $i_{1}: \mathscr{A} \rightarrow \mathscr{C}$ and $j^{\natural}: \mathscr{C} \rightarrow \mathscr{B}$ be the functors defined by

$$
\begin{array}{ll}
i_{!}(A)=\left(A, F A, 1_{F A}\right) & i_{!}(\alpha)=(\alpha, F \alpha) \\
j^{\natural}(A, B, f)=\text { coker } f & j^{\natural}(\alpha, \beta)=\bar{\beta}
\end{array}
$$

where $\bar{\beta}$ : coker $f \rightarrow$ coker $f^{\prime}$ is induced from $\beta$.

Lemma $3.2 i_{\text {! }}$ is a left adjoint of $i^{-1}, j^{\natural}$ is a left adjoint of $j$, and

$$
i^{-1} i_{!} \simeq \operatorname{Id}_{\mathscr{A}} \quad j^{-1} i_{!}=F \quad j^{\natural} i_{!}=j^{\natural} i_{*}=0 \quad j^{\natural} j_{!} \simeq \operatorname{Id}_{\mathscr{B}}
$$

Proof The adjunctions follow by considering the commutative diagrams
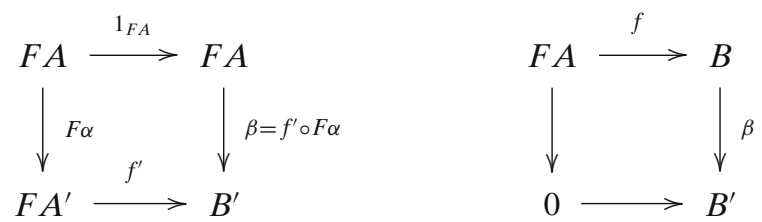

and noting that the commutativity of the right diagram implies that $\beta$ factors uniquely through coker $f$. The other relations are straightforward.

Remark 3.3 The diagram

$$
\mathscr{A} \times \mathscr{B} \underset{\left(i^{-1}, j^{-1}\right)}{\stackrel{\left(i_{1}, j_{!}\right)}{\longrightarrow}} \mathscr{C} \underset{\left(i_{*}, j_{!}\right)}{\stackrel{\left(i^{-1}, j^{\sharp}\right)}{\longrightarrow}} \mathscr{A} \times \mathscr{B}
$$

is a special case of the one in [8, p. 7], viewing $\mathscr{C}$ as a trivial extension of $\mathscr{A} \times \mathscr{B}$.

Proposition 3.4 Let $P$ be a projective object of $\mathscr{A}$ and $T_{\mathscr{B}}$ be a rigid object of $\mathscr{B}$ such that $\operatorname{Ext}_{\mathscr{B}}^{n}\left(F P, T_{\mathscr{B}}\right)=0$ for all $n>0$. Assume that $F P \in \mathrm{ob}(\mathscr{B})$ has a projective resolution in $\mathscr{B}$ and consider $T=(P, 0,0) \oplus\left(0, T_{\mathscr{B}}, 0\right)[1] \in \mathscr{D}^{b}(\mathscr{C})$. Then $\operatorname{Hom}_{\mathscr{D}^{b}(\mathscr{C})}(T, T[n])=0$ for $n \neq 0$ and

$$
\operatorname{End}_{\mathscr{D}^{b}(\mathscr{C})}(T) \simeq\left(\begin{array}{cc}
\operatorname{End}_{\mathscr{B}}\left(T_{\mathscr{B}}\right) & \operatorname{Hom}_{\mathscr{B}}\left(F P, T_{\mathscr{B}}\right) \\
0 & \operatorname{End}_{\mathscr{A}}(P)
\end{array}\right)
$$

where the bimodule structure on $\operatorname{Hom}_{\mathscr{B}}\left(F P, T_{\mathscr{B}}\right)$ is given by left composition with $\operatorname{End}_{\mathscr{B}}\left(T_{\mathscr{B}}\right)$ and right composition with $\mathrm{End}_{\mathscr{B}}(F P)$ through the natural map $\operatorname{End}_{\mathscr{A}}(P) \rightarrow \operatorname{End}_{\mathscr{B}}(F P)$. 
Proof Since $i_{\text {! }}$ is a left adjoint of an exact functor, it takes projective objects of $\mathscr{A}$ to projective objects of $\mathscr{C}$. Hence $i_{1} P=\left(P, F P, 1_{F P}\right)$ is projective and we can apply Proposition 2.4 for $i_{!} P$ and $T_{\mathscr{B}}$. As $(P, 0,0)=i_{*} i^{-1} i_{!} P$ and $\left(0, T_{\mathscr{B}}, 0\right)=j ! T_{\mathscr{B}}$, we only need to show the isomorphism

$$
\operatorname{Ext}_{\mathscr{C}}^{1}\left((P, 0,0),\left(0, T_{\mathscr{B}}, 0\right)\right) \simeq \operatorname{Hom}_{\mathscr{B}}\left(F P, T_{\mathscr{B}}\right)
$$

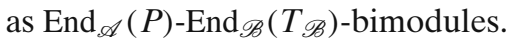

Indeed, let

$$
\cdots \rightarrow Q^{2} \rightarrow Q^{1} \rightarrow F P \rightarrow 0
$$

be a projective resolution of $F P$. Then $(P, 0,0)$ is quasi-isomorphic to the complex

$$
\cdots \rightarrow j_{!} Q^{2} \rightarrow j_{!} Q^{1} \rightarrow i_{!} P \rightarrow 0 \rightarrow \ldots
$$

whose terms are projective since $j !$ is a left adjoint of an exact functor. Therefore $\operatorname{Ext}_{\mathscr{C}}^{1}\left((P, 0,0),\left(0, T_{\mathscr{B}}, 0\right)\right)$ can be identified with the morphisms, up to homotopy, between the complexes

$$
\begin{aligned}
& \cdots \longrightarrow j_{!} Q^{2} \longrightarrow j_{!} Q^{1} \longrightarrow i_{1} P \longrightarrow 0 \longrightarrow \cdots \\
& \cdots \longrightarrow 0 \longrightarrow{ }_{1} T_{\mathscr{B}} \longrightarrow 0 \longrightarrow
\end{aligned}
$$

By Lemma 3.2, $\operatorname{Hom}_{\mathscr{C}}\left(i_{!} P, j_{!} T_{\mathscr{B}}\right)=\operatorname{Hom}_{\mathscr{A}}\left(P, i^{-1} j_{!} T_{\mathscr{B}}\right)=0$, thus any homotopy between these complexes vanishes, and the morphism space equals $\operatorname{ker}\left(\operatorname{Hom}_{\mathscr{C}}\left(j ! Q^{1}, j_{!} T_{\mathscr{B}}\right) \rightarrow \operatorname{Hom}_{\mathscr{C}}\left(j ! Q^{2}, j_{!} T_{\mathscr{B}}\right)\right)$. Using the fact that $j$ ! is fully faithful and applying the functor $\operatorname{Hom}_{\mathscr{B}}\left(-, T_{\mathscr{B}}\right)$ on the exact sequence (3.2), we get that the morphism space equals $\operatorname{Hom}_{\mathscr{B}}\left(F P, T_{\mathscr{B}}\right)$, as desired.

Under this identification, the left action of $\operatorname{End}_{\mathscr{B}}\left(T_{\mathscr{B}}\right) \simeq \operatorname{End}_{\mathscr{C}}\left(j_{!} T_{\mathscr{B}}\right)$ is given by left composition. As for the right action of $\operatorname{End}_{\mathscr{A}}(P)$, observe that any $\alpha \in \operatorname{End}_{\mathscr{A}}(P)$ extends uniquely to an endomorphism in the homotopy category

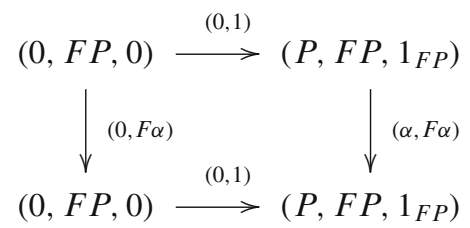

which determines a unique endomorphism, in the homotopy category, of the top complex of Eq. 3.3.

Remark 3.5 When the functor $F: \mathscr{A} \rightarrow \mathscr{B}$ admits a right adjoint $G: \mathscr{B} \rightarrow \mathscr{A}$, the comma category ( $F \downarrow \mathrm{Id}$ ) is equivalent to the comma category (Id $\downarrow G$ ) corresponding to the pair $\mathscr{A} \stackrel{\text { Id }}{\rightarrow} \mathscr{A} \stackrel{G}{\leftarrow} \mathscr{B}$. In this case, one can define also a right adjoint $i^{!}$of $i_{*}$ and a right adjoint $j_{*}$ of $j^{-1}$, and we end up with the eight functors $\left(i_{!}, i^{-1}, i_{*}, i^{!}\right)$ and $\left(j^{\natural}, j_{!}, j^{-1}, j_{*}\right)$. The bimodule $\operatorname{Hom}_{\mathscr{B}}\left(F P, T_{\mathscr{B}}\right)$ in Proposition 3.4 can then be identified with $\operatorname{Hom}_{\mathscr{A}}\left(P, G T_{\mathscr{B}}\right)$. 


\section{Application to Triangular Matrix Rings}

\subsection{Triangular Matrix Rings}

Let $R$ and $S$ be rings, and let ${ }_{R} M_{S}$ be an $S$ - $R$-bimodule. Let $\Lambda$ be the triangular matrix ring

$$
\Lambda=\left(\begin{array}{cc}
R & M \\
0 & S
\end{array}\right)=\left\{\left(\begin{array}{cc}
r & m \\
0 & s
\end{array}\right): r \in R, s \in S, m \in M\right\}
$$

where the ring structure is induced by the ordinary matrix operations.

For a ring $R$, denote the category of right $R$-modules by $\operatorname{Mod} R$. The functor $-\otimes$ $M: \operatorname{Mod} R \rightarrow \operatorname{Mod} S$ is additive and right exact, hence the corresponding comma category $\left(-\otimes M \downarrow \operatorname{Id}_{\text {Mod } S}\right)$ is abelian.

Lemma 4.1 [1, III.2] The category $\operatorname{Mod} \Lambda$ is equivalent to the comma category $(-\otimes$ $\left.M \downarrow \operatorname{Id}_{\operatorname{Mod} S}\right)$.

Proof One verifies that by sending a triple $\left(X_{R}, Y_{S}, f: X \otimes M \rightarrow Y\right)$ to the $\Lambda$ module $X \oplus Y$ defined by

$$
\left(\begin{array}{ll}
x & y
\end{array}\right)\left(\begin{array}{cc}
r & m \\
0 & s
\end{array}\right)=(x r f(x \otimes m)+y s)
$$

and sending a morphism $(\alpha, \beta):(X, Y, f) \rightarrow\left(X^{\prime}, Y^{\prime}, f^{\prime}\right)$ to $\alpha \oplus \beta: X \oplus Y \rightarrow X^{\prime} \oplus$ $Y^{\prime}$, we get a functor $\left(-\otimes M \downarrow \operatorname{Id}_{\operatorname{Mod} S}\right) \rightarrow \operatorname{Mod} \Lambda$ which is an equivalence of categories.

Corollary 4.2 There exist gluing data

$$
\operatorname{Mod} R \underset{i^{-1}}{\stackrel{i_{*}}{\gtrless}} \operatorname{Mod} \Lambda \underset{j !}{\stackrel{j^{-1}}{\rightleftarrows}} \operatorname{Mod} S
$$

The functors occurring in Corollary 4.2 can be described explicitly. Let

$$
e_{R}=\left(\begin{array}{ll}
1 & 0 \\
0 & 0
\end{array}\right), \quad e_{S}=\left(\begin{array}{ll}
0 & 0 \\
0 & 1
\end{array}\right)
$$

Using Eq. 4.2, observe that for a $\Lambda$-module $Z_{\Lambda}$,

$$
\left(i^{-1} Z\right)_{R}=Z e_{R} \quad\left(j^{-1} Z\right)_{S}=Z e_{S}
$$

where $r$ acts on $i^{-1} Z$ via $\left(\begin{array}{ll}r & 0 \\ 0 & 0\end{array}\right)$ and $s$ acts on $j^{-1} Z$ via $\left(\begin{array}{ll}0 & 0 \\ 0 & s\end{array}\right)$. The morphism $\left(i^{-1} Z\right) \otimes$ $M \rightarrow j^{-1} Z$ is obtained by considering the actions of $\left(\begin{array}{ll}0 & m \\ 0 & 0\end{array}\right), m \in M$, and the map $Z \mapsto\left(i^{-1} Z, j^{-1} Z,\left(i^{-1} Z\right) \otimes M \rightarrow j^{-1} Z\right)$ defines a functor which is an inverse to the equivalence of categories constructed in the proof of Lemma 4.1.

Conversely, for an $R$-module $X_{R}$ and $S$-module $Y_{S}$, we have $\left(i_{*} X\right)_{\Lambda}=X$ and $(j ! Y)_{\Lambda}=Y$ where $\left(\begin{array}{ll}r & m \\ 0 & s\end{array}\right)$ acts on $X$ via $r$ and on $Y$ via $s$. 
Lemma 4.3 The image of $\Lambda_{\Lambda}$ in the comma category equals $\left(R, M, 1_{M}\right) \oplus(0, S, 0)$.

Proof Use Eq. 4.3 and

$$
\Lambda\left(\begin{array}{ll}
1 & 0 \\
0 & 0
\end{array}\right)=\left(\begin{array}{ll}
R & 0 \\
0 & 0
\end{array}\right), \quad \quad \Lambda\left(\begin{array}{ll}
0 & 0 \\
0 & 1
\end{array}\right)=\left(\begin{array}{ll}
0 & M \\
0 & S
\end{array}\right) .
$$

Remark 4.4 Since $-\otimes M$ admits a right adjoint $\operatorname{Hom}(M,-)$, we are in the situation of Remark 3.5 and there are eight functors $\left(i_{!}, i^{-1}, i_{*}, i^{!}\right)$and $\left(j^{\natural}, j_{!}, j^{-1}, j_{*}\right)$. For the convenience of the reader, we now describe them as standard functors $\otimes$ and Hom involving idempotents, see also [6, Section 2] and [17, Proposition 2.17].

If $A$ is a ring and $e \in A$ is an idempotent, the functor

$$
\operatorname{Hom}_{A}(e A,-)=-\otimes_{A} A e: \operatorname{Mod} A \rightarrow \operatorname{Mod} e A e
$$

admits a left adjoint $-\otimes_{e A e} e A$ and a right adjoint $\operatorname{Hom}_{e A e}(A e,-)$. By taking $A=\Lambda$ and $e=e_{R}$ we get the three functors $\left(i_{!}, i^{-1}, i_{*}\right)$. Similarly, $e=e_{S}$ gives $\left(j_{!}, j^{-1}, j_{*}\right)$.

In addition, the natural inclusion functor

$$
\operatorname{Hom}_{A / A e A}(A / A e A,-)=-\otimes_{A / A e A} A / A e A: \operatorname{Mod} A / A e A \rightarrow \operatorname{Mod} A
$$

admits a left adjoint $-\otimes_{A} A / A e A$ and a right adjoint $\operatorname{Hom}_{A}(A / A e A,-)$. By taking $A=\Lambda$ and $e=e_{R}$, observing that $e_{S} \Lambda e_{R}=0$, we get the three functors $\left(j^{\natural}, j_{!}, j^{-1}\right)$. For $e=e_{S}$ we get $\left(i^{-1}, i_{*}, i^{!}\right)$.

\subsection{The Main Theorem}

For a ring $\Lambda$, denote by $\mathscr{D}^{b}(\Lambda)$ the bounded derived category of $\operatorname{Mod} \Lambda$, and by per $\Lambda$ its full subcategory of complexes quasi-isomorphic to perfect complexes, that is, bounded complexes of finitely generated projective $\Lambda$-modules.

For a complex $T \in \mathscr{D}^{b}(\Lambda)$, denote by $\langle\operatorname{add} T\rangle$ the smallest full triangulated subcategory of $\mathscr{D}^{b}(\Lambda)$ containing $T$ and closed under forming direct summands. Recall that $T$ is a tilting complex if $\langle$ add $T\rangle=\operatorname{per} \Lambda$ and $\operatorname{Hom}_{\mathscr{D}^{b}(\Lambda)}(T, T[n])=0$ for all integers $n \neq 0$. If, moreover, $H^{n}(T)=0$ for all $n \neq 0$, we call $T$ a tilting module and identify it with the module $H^{0}(T)$.

Theorem 4.5 Let $R, S$ be rings and $T_{S}$ a tilting $S$-module. Let ${ }_{R} M_{S}$ be an $S$ - $R$ bimodule such that as an $S$-module, $M_{S} \in \operatorname{per} S$ and $\operatorname{Ext}_{S}^{n}\left(M_{S}, T_{S}\right)=0$ for all $n>0$. Then the triangular matrix rings

$$
\Lambda=\left(\begin{array}{cc}
R & M \\
0 & S
\end{array}\right) \quad \text { and } \quad \widetilde{\Lambda}=\left(\begin{array}{cc}
\operatorname{End}_{S}\left(T_{S}\right) & \operatorname{Hom}_{S}\left(M, T_{S}\right) \\
0 & R
\end{array}\right)
$$

are derived equivalent.

Proof For simplicity, we shall identify $\operatorname{Mod} \Lambda$ with the corresponding comma category. We will show that $T=(R, \underset{\sim}{0}, 0) \oplus\left(0, T_{S}, 0\right)[1]$ is a tilting complex in $\mathscr{D}^{b}(\Lambda)$ whose endomorphism ring equals $\widetilde{\Lambda}$. 
Applying Proposition 3.4 for the projective $R$-module $R$ and the rigid $S$ module $T_{S}$, noting that $F R=M_{S}$ and $\operatorname{Ext}_{S}^{n}\left(M_{S}, T_{S}\right)=0$ for $n>0$, we see that $\operatorname{Hom}_{\mathscr{D}^{b}(\Lambda)}(T, T[n])=0$ for all $n \neq 0$ and moreover $\operatorname{End}_{\mathscr{D}^{b}(\Lambda)}(T) \simeq \widetilde{\Lambda}$.

It remains to show that $\langle$ add $T\rangle=\operatorname{per} \Lambda$. First, we show that $T \in \operatorname{per} \Lambda$. Observe that $j_{!}(\operatorname{per} S) \subseteq \operatorname{per} \Lambda$, since $j$ ! is an exact functor which takes projectives to projectives and $j ! S=(0, S, 0)$ is a direct summand of $\Lambda$. Hence in the short exact sequence

$$
0 \rightarrow(0, M, 0) \rightarrow\left(R, M, 1_{M}\right) \rightarrow(R, 0,0) \rightarrow 0
$$

we have that $(0, M, 0) \in \operatorname{per} \Lambda$ by the assumption that $M_{S} \in \operatorname{per} S$, and $\left(R, M, 1_{M}\right) \in$ per $\Lambda$ as a direct summand of $\Lambda$. Therefore $(R, 0,0) \in$ per $\Lambda$. In addition, $\left(0, T_{S}, 0\right) \in$ per $\Lambda$ by the assumption $T_{S} \in \operatorname{per} S$, hence $T$ is isomorphic in $\mathscr{D}^{b}(\Lambda)$ to a perfect complex.

Second, in order to prove that $\langle$ add $T\rangle=\operatorname{per} \Lambda$ it is enough to show that $\Lambda \in$ $\langle$ add $T\rangle$. Indeed, since $\left(0, T_{S}, 0\right)[1]$ is a summand of $T$, by the exactness of $j$ ! and our assumption that $\left\langle\right.$ add $\left.T_{S}\right\rangle=\operatorname{per} S$, we have that $(0, S, 0) \in\langle\operatorname{add} T\rangle$ and $(0, M, 0) \in$ $\langle$ add $T\rangle$. Since $(R, 0,0)$ is a summand of $T$, by invoking again the short exact sequence (4.4) we see that $\left(R, M, 1_{M}\right) \in\langle\operatorname{add} T\rangle$, hence $\Lambda \in\langle\operatorname{add} T\rangle$.

Therefore $T$ is a tilting complex in $\mathscr{D}^{b}(\Lambda)$, and by [19] (see also [12, (1.4)]), the rings $\Lambda$ and $\tilde{\Lambda} \simeq \operatorname{End}_{\mathscr{D}^{b}(\Lambda)}(T)$ are derived equivalent.

Remark 4.6 The assumption that $T_{S}$ is a tilting module implies that the rings $S$ and $\operatorname{End}_{S}\left(T_{S}\right)$ are derived equivalent.

Remark 4.7 When the tilting module $T_{S}$ is also injective, it is enough to assume that $M_{S} \in \operatorname{per} S$.

\subsection{Applications}

Let $S$ be an Artin algebra over an Artinian commutative ring $k$, and let $\bmod S$ be the category of finitely generated right $S$-modules. Let $D: \operatorname{Mod} S \rightarrow \operatorname{Mod} S^{o p}$ be the functor defined by $D=\operatorname{Hom}_{k}(-, J)$, where $J$ is an injective envelope of the direct sum of all the non-isomorphic simple modules of $k$. Recall that $D$ restricts to a duality $D: \bmod S \rightarrow \bmod S^{o p}$. Applying it on the bimodule ${ }_{S} S_{S}$, we get the bimodule ${ }_{S} D S_{S}=$ $\operatorname{Hom}_{k}(S, J)$.

Lemma 4.8 Let $R$ be a ring and ${ }_{R} M_{S}$ a bimodule. Then ${ }_{S} D M_{R} \simeq \operatorname{Hom}_{S}\left({ }_{R} M_{S},{ }_{S} D S_{S}\right)$ as $R$-S-bimodules.

Proof By the standard adjunctions,

$$
\operatorname{Hom}_{S}\left({ }_{R} M_{S}, \operatorname{Hom}_{k}\left(S_{S}, J\right)\right) \simeq \operatorname{Hom}_{k}\left({ }_{R} M_{S} \otimes_{S} S_{S}, J\right)=\operatorname{Hom}_{k}\left({ }_{R} M_{S}, J\right) .
$$

It follows that $D=\operatorname{Hom}_{S}\left(-, D S_{S}\right)$, hence $D S_{S}$ is an injective object in Mod $S$. We denote by gl.dim $S$ the global dimension of $\bmod S$. 
Theorem 4.9 Let $R$ be a ring, $S$ an Artin algebra with gl.dim $S<\infty$ and ${ }_{R} M_{S}$ an $S$ $R$-bimodule which is finitely generated as an $S$-module. Then the triangular matrix rings

$$
\Lambda=\left(\begin{array}{cc}
R & M \\
0 & S
\end{array}\right) \text { and } \tilde{\Lambda}=\left(\begin{array}{cc}
S & D M \\
0 & R
\end{array}\right)
$$

are derived equivalent, where $D$ is the duality on $\bmod S$.

Proof The module $D S_{S}$ is injective in $\operatorname{Mod} S$ and any module in $\bmod S$ has an injective resolution with terms that are summands of finite direct sums of $D S$. Since gl.dim $S<\infty$, such a resolution is finite, hence $\langle$ add $D S\rangle=\operatorname{per} S$ and $M \in \operatorname{per} S$ for any $M \in \bmod S$.

Therefore the assumptions of Theorem 4.5 are satisfied for $T_{S}=D S$ (see also Remark 4.7), and it remains to show that $\operatorname{End}_{S}\left(T_{S}\right)=S$ and $\operatorname{Hom}_{S}\left(M, T_{S}\right) \simeq{ }_{S} D M_{R}$ (as bimodules). This follows by the Lemma 4.8 applied for the bimodules ${ }_{S} D S_{S}$ and ${ }_{R} M_{S}$.

Remark 4.10 Under the assumptions of Theorem 4.9, when $R$ is also an Artin $k$ algebra and $k$ acts centrally on $M$, the rings $\Lambda$ and $\tilde{\Lambda}$ are Artin algebras and the derived equivalence in the theorem implies that $\mathscr{D}^{b}(\bmod \Lambda) \simeq \mathscr{D}^{b}(\bmod \tilde{\Lambda})$.

Moreover, by using the duality $D$, one sees that Theorem 4.9 is true for two Artin algebras $R$ and $S$ and a bimodule ${ }_{R} M_{S}$ on which $k$ acts centrally under the weaker assumptions that $M$ is finitely generated over $k$ and at least one of gl.dim $R, \operatorname{gl} \cdot \operatorname{dim} S$ is finite.

By taking $T_{S}=S$ in Theorem 4.5, we get the following corollary.

Corollary 4.11 Let $R$, $S$ be rings and ${ }_{R} M_{S}$ an $S$ - $R$-bimodule such that as an $S$-module, $M_{S} \in \operatorname{per} S$ and $\operatorname{Ext}_{S}^{n}\left(M_{S}, S\right)=0$ for all $n>0$. Then the triangular matrix rings

$$
\Lambda=\left(\begin{array}{cc}
R & M \\
0 & S
\end{array}\right) \text { and } \tilde{\Lambda}=\left(\begin{array}{cc}
S \operatorname{Hom}_{S}(M, S) \\
0 & R
\end{array}\right)
$$

are derived equivalent.

Remark 4.12 The conditions of Corollary 4.11 hold when the ring $S$ is self-injective, that is, $S$ is injective as a (right) module over itself, and ${ }_{R} M_{S}$ is finitely generated projective as an $S$-module. In particular, this applies when $S$ is a semi-simple ring and $M$ is finitely generated as an $S$-module.

Remark 4.13 Recall that for a ring $R$, a division ring $S$ and a bimodule ${ }_{S} N_{R}$ which is finite dimensional as a left $S$-vector space, the one-point extension $R[N]$ and the one-point coextension $[N] R$ of $R$ by $N$ are defined as the triangular matrix rings

$$
R[N]=\left(\begin{array}{cc}
S_{S} N_{R} \\
0 & R
\end{array}\right) \quad[N] R=\left(\begin{array}{cc}
R & D N_{S} \\
0 & S
\end{array}\right)
$$

where $D=\operatorname{Hom}_{S}(-, S)$ is the duality on $\bmod S$. By taking $M=D N$ in the preceding remark, we see that the rings $R[N]$ and $[N] R$ are derived equivalent. Compare this with the construction of "reflection with respect to an idempotent" in [20]. 


\section{Concluding Remarks}

\subsection{Repetitive Algebras}

In the specific case of Artin algebras, another approach to the connection between a triangular matrix algebra $\Lambda$ and its mate $\widetilde{\Lambda}$ involves the use of repetitive algebras, as outlined below.

Let $\Lambda$ be an Artin algebra over a commutative Artinian ring $k$ and let $D: \bmod k \rightarrow$ $\bmod k$ be the duality. Recall that the repetitive algebra $\widehat{\Lambda}$ of $\Lambda$, introduced in [11], is the algebra (without unit) of matrices of the form

$$
\widehat{\Lambda}=\left(\begin{array}{cccc}
\ddots & D \Lambda_{i-1} & 0 & \\
0 & \Lambda_{i} & D \Lambda_{i} & 0 \\
& 0 & \Lambda_{i+1} & D \Lambda_{i+1} \\
& & 0 & \ddots
\end{array}\right)
$$

where $\Lambda_{i}=\Lambda, D \Lambda_{i}=D \Lambda$ for $i \in \mathbb{Z}$, and only a finite number of entries are nonzero. The multiplication is defined by the canonical maps $\Lambda \otimes_{\Lambda} D \Lambda \rightarrow D \Lambda, D \Lambda \otimes_{\Lambda} \Lambda \rightarrow$ $D \Lambda$ induced by the bimodule structure on $D \Lambda$, and the zero map $D \Lambda \otimes_{\Lambda} D \Lambda \rightarrow 0$.

When $\Lambda$ is a triangular matrix algebra, one can write

$$
\Lambda=\left(\begin{array}{ll}
R & M \\
0 & S
\end{array}\right) \quad D \Lambda=\left(\begin{array}{cr}
D R & 0 \\
D M & D S
\end{array}\right)
$$

and a direct calculation shows that the maps $\Lambda \otimes D \Lambda \rightarrow D \Lambda$ and $D \Lambda \otimes \Lambda \rightarrow D \Lambda$ are given by multiplication of the above matrices, under the convention that $M \otimes_{S} D S \rightarrow 0$ and $D R \otimes_{R} M \rightarrow 0$.

As for the mate $\tilde{\Lambda}$, we have

$$
\widetilde{\Lambda}=\left(\begin{array}{cc}
S & D M \\
0 & R
\end{array}\right) \quad D \widetilde{\Lambda}=\left(\begin{array}{cc}
D S & 0 \\
M & D R
\end{array}\right)
$$

therefore the repetitive algebras of $\Lambda$ and its mate $\tilde{\Lambda}$ have the form

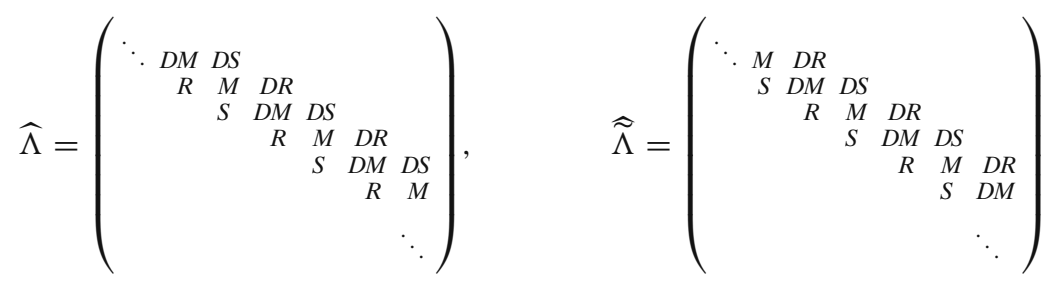

and are thus clearly seen to be isomorphic.

When $k$ is a field and both algebras $R$ and $S$ have finite global dimension, this can be combined with Happel's Theorem [9, (II.4.9)] to deduce that $\Lambda$ and its mate $\widetilde{\Lambda}$ are derived equivalent.

Note, however, that for the derived equivalence between $\Lambda$ and $\tilde{\Lambda}$ to hold, it is enough to assume that only one of $R, S$ has finite global dimension (see Remark 4.10). 
Moreover, while the repetitive algebras of $\Lambda$ and $\tilde{\Lambda}$ are always isomorphic, in the case where none of $R, S$ have finite global dimension, the algebras $\Lambda$ and $\widetilde{\Lambda}$ may not be derived equivalent, see Example 5.3 below.

\subsection{Grothendieck Groups}

In this subsection, $k$ denotes an algebraically closed field. Let $\Lambda$ be a finite dimensional $k$-algebra and let $P_{1}, \ldots, P_{n}$ be a complete collection of the non-isomorphic indecomposable projectives in mod $\Lambda$. The Cartan matrix of $\Lambda$ is the $n \times n$ integer matrix defined by $C_{i j}=\operatorname{dim}_{k} \operatorname{Hom}\left(P_{i}, P_{j}\right)$.

The Grothendieck group $K_{0}$ (per $\Lambda$ ) of the triangulated category per $\Lambda$ can be viewed as a free abelian group on the generators $\left[P_{1}\right], \ldots,\left[P_{n}\right]$, and the Euler form

$$
\langle K, L\rangle=\sum_{r \in \mathbb{Z}}(-1)^{r} \operatorname{dim}_{k} \operatorname{Hom}_{\mathscr{D}^{b}(\Lambda)}(K, L[r])
$$

on per $\Lambda$ induces a bilinear form on $K_{0}(\operatorname{per} \Lambda)$ whose matrix with respect to that basis equals the Cartan matrix.

It is well known that a derived equivalence of two algebras $\Lambda$ and $\Lambda^{\prime}$ induces an equivalence of the triangulated categories per $\Lambda$ and per $\Lambda^{\prime}$, and hence an isometry of their Grothendieck groups preserving the Euler forms. We now consider the consequences of the derived equivalence of Theorem 4.9 (when $R$ and $S$ are finite dimensional $k$-algebras) for the corresponding Grothendieck groups.

For simplicity, assume that $\Lambda$ is basic. In this case, there exist primitive orthogonal idempotents $\left\{e_{1}, \ldots, e_{n}\right\}$ in $\Lambda$ such that $P_{i} \simeq e_{i} \Lambda$ for $1 \leq i \leq n$. Therefore by the isomorphisms $\operatorname{Hom}_{\Lambda}\left(e_{i} \Lambda, N\right) \simeq N e_{i}$ of $k$-spaces for any $\Lambda$-module $N_{\Lambda}$, we get that $C_{i j}=\operatorname{dim}_{k} e_{j} \Lambda e_{i}$.

Lemma 5.1 Let $R, S$ be basic, finite dimensional k-algebras, and let ${ }_{R} M_{S}$ be a finite dimensional $S$-R-bimodule. Then the Cartan matrix $C_{\Lambda}$ of the corresponding triangular matrix algebra $\Lambda$ is of the form

$$
C_{\Lambda}=\left(\begin{array}{cc}
C_{R} & 0 \\
C_{M} & C_{S}
\end{array}\right)
$$

where $C_{R}, C_{S}$ are the Cartan matrices of $R, S$.

Proof Let $e_{1}, \ldots, e_{n}$ and $f_{1}, \ldots, f_{m}$ be complete sets of primitive orthogonal idempotents in $R$ and in $S$. Let $\bar{e}_{i}=e_{i}\left(\begin{array}{ll}1 & 0 \\ 0 & 0\end{array}\right)$ and $\bar{f}_{j}=f_{j}\left(\begin{array}{ll}0 & 0 \\ 0 & 1\end{array}\right)$. Then $\bar{e}_{1}, \ldots, \bar{e}_{n}, \bar{f}_{1}, \ldots, \bar{f}_{m}$ is a complete set of primitive orthogonal idempotents of $\Lambda$ and the result follows by computing the dimensions of $\bar{e}_{i} \Lambda \bar{e}_{i^{\prime}}, \bar{e}_{i} \Lambda \bar{f}_{j}, \bar{f}_{j} \Lambda \bar{e}_{i}$ and $\bar{f}_{j} \Lambda \bar{f}_{j^{\prime}}$. In particular, $\left(C_{M}\right)_{j i}=$ $\operatorname{dim}_{k} e_{i} M f_{j}$.

Since $\operatorname{dim}_{k} f_{j} D M e_{i}=\operatorname{dim}_{k} e_{i} M f_{j}$, we get by Lemma 5.1 that the Cartan matrices of $\Lambda$ and its mate $\widetilde{\Lambda}$ are

$$
C_{\Lambda}=\left(\begin{array}{cc}
C_{R} & 0 \\
C_{M} & C_{S}
\end{array}\right) \quad C_{\widetilde{\Lambda}}=\left(\begin{array}{cc}
C_{S} & 0 \\
C_{M}^{t} & C_{R}
\end{array}\right) .
$$

When at least one of $R$ and $S$ has finite global dimension, the derived equivalence of Theorem 4.9 implies that $C_{\Lambda}$ and $C_{\widetilde{\Lambda}}$ represent the same bilinear form, hence they 
are congruent over $\mathbb{Z}$, that is, there exists an invertible matrix $P$ over $\mathbb{Z}$ such that $P^{t} C_{\Lambda} P=C_{\widetilde{\Lambda}}$.

One can also show this congruence directly at the level of matrices, as follows.

Lemma 5.2 Let $K$ be a commutative ring. Let $A \in M_{n \times n}(K)$ be a square matrix, $B \in \mathrm{GL}_{m}(K)$ an invertible square matrix and $C \in M_{m \times n}(K)$. Then there exists $P \in$ $\mathrm{GL}_{n+m}(K)$ such that

$$
P^{t}\left(\begin{array}{ll}
A & 0 \\
C & B
\end{array}\right) P=\left(\begin{array}{ll}
B & 0 \\
C^{t} & A
\end{array}\right)
$$

Proof Take $P=\left(\begin{array}{cc}0 & I_{n} \\ -B^{-1} B^{t} & -B^{-1} C\end{array}\right)$. Then

$$
\begin{aligned}
& P^{t}\left(\begin{array}{cc}
A & 0 \\
C & B
\end{array}\right) P=\left(\begin{array}{c}
0-B B^{-t} \\
I_{n}-C^{t} B^{-t}
\end{array}\right)\left(\begin{array}{cc}
A & 0 \\
C & B
\end{array}\right)\left(\begin{array}{cc}
0 & I_{n} \\
-B^{-1} B^{t} & -B^{-1} C
\end{array}\right) \\
& =\left(\begin{array}{c}
0-B B^{-t} \\
I_{n}-C^{t} B^{-t}
\end{array}\right)\left(\begin{array}{cc}
0 & A \\
-B^{t} & 0
\end{array}\right)=\left(\begin{array}{cc}
B & 0 \\
C^{t} & A
\end{array}\right)
\end{aligned}
$$

Note that one could also take $P=\left(\begin{array}{c}-A^{-t} C^{t}-A^{-t} A \\ I_{m}\end{array} \begin{array}{l}0\end{array}\right)$, hence it is enough to assume that at least one of $A$ and $B$ is invertible.

The conclusion of the lemma is false if one does not assume that at least one of the matrices $A, B$ is invertible over $K$. This can be used to construct triplets consisting of two finite dimensional algebras $R, S$ (necessarily of infinite global dimension) and a bimodule $M$ such that the triangular matrix algebra $\Lambda$ and its mate $\widetilde{\Lambda}$ are not derived equivalent.

Example 5.3 Let $R=k[x] /\left(x^{2}\right), S=k[y] /\left(y^{3}\right)$ and $M=k$ with $x$ and $y$ acting on $k$ as zero. Then the triangular matrix algebras

$$
\Lambda=\left(\begin{array}{cc}
k[x] /\left(x^{2}\right) & k \\
0 & k[y] /\left(y^{3}\right)
\end{array}\right) \quad \tilde{\Lambda}=\left(\begin{array}{cc}
k[y] /\left(y^{3}\right) & k \\
0 & k[x] /\left(x^{2}\right)
\end{array}\right)
$$

are not derived equivalent, since one can verify that their Cartan matrices

$$
C_{\Lambda}=\left(\begin{array}{ll}
2 & 0 \\
1 & 3
\end{array}\right) \quad C_{\widetilde{\Lambda}}=\left(\begin{array}{ll}
3 & 0 \\
1 & 2
\end{array}\right)
$$

are not congruent over $\mathbb{Z}$. Note that despite the fact that $R$ and $S$ are self-injective, Corollary 4.11 cannot be used since $M$ does not have a finite projective resolution.

\subsection{Trivial Extensions}

Triangular matrix rings are special cases of trivial extensions [1, p. 78]. Indeed, if $R, S$ are rings and ${ }_{R} M_{S}$ is a bimodule, the corresponding triangular matrix ring is isomorphic to the trivial extension $A \ltimes M$ where $A=R \times S$ and $M$ is equipped with an $A$-bimodule structure via $(r, s) m=r m$ and $m(r, s)=m s$. 
We remark that even when $A$ is a finite dimensional $k$-algebra of finite global dimension and $M$ is a finite dimensional $A$-bimodule, the trivial extension algebras $A \ltimes M$ and $A \ltimes D M$ are generally not derived equivalent, so that the derived equivalence in Theorem 4.9 is a special feature of triangular matrix rings.

Example 5.4 Let $A=k Q$ where $Q$ is the quiver $\bullet_{1} \longrightarrow \bullet_{2}$ and let $M$ be the $k Q$-bimodule corresponding to the following commutative diagram of vector spaces

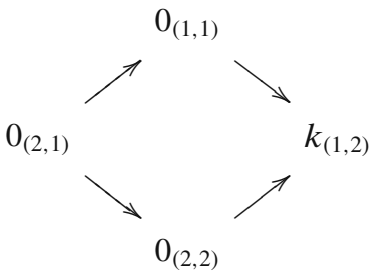

Then $A \ltimes M$ is the path algebra of the quiver $\bullet \rightrightarrows \bullet$ while $A \ltimes D M$ is the path algebra of $\bullet \rightleftarrows \bullet$ modulo the compositions of the arrows being zero. These two algebras are not derived equivalent since $\operatorname{gl} \operatorname{dim}(A \ltimes M)=1$ while $\operatorname{gl} \cdot \operatorname{dim}(A \ltimes D M)=\infty$.

Acknowledgements I am grateful to B. Keller for discussions of a preliminary version of [14] which led to this current paper.

Open Access This article is distributed under the terms of the Creative Commons Attribution Noncommercial License which permits any noncommercial use, distribution, and reproduction in any medium, provided the original author(s) and source are credited.

\section{References}

1. Auslander, M., Reiten, I., Smalø, S.O.: Representation theory of Artin algebras. Cambridge Studies in Advanced Mathematics, vol. 36. Cambridge University Press, Cambridge (1995)

2. Barot, M., Lenzing, H.: One-point extensions and derived equivalence. J. Algebra 264(1), 1-5 (2003)

3. Beŭlinson, A.A., Bernstein, J., Deligne, P.: Faisceaux pervers. In: Analysis and Topology on Singular Spaces, I (Luminy, 1981), Astérisque, vol. 100, pp. 5-171. Soc. Math. France, Paris (1982)

4. Chase, S.U.: A generalization of the ring of triangular matrices. Nagoya Math. J. 18, 13-25 (1961)

5. Cline, E., Parshall, B., Scott, L.: Algebraic stratification in representation categories. J. Algebra 117(2), 504-521 (1988)

6. Cline, E., Parshall, B., Scott, L.: Finite-dimensional algebras and highest weight categories. J. Reine Angew. Math. 391, 85-99 (1988)

7. Fields, K.L.: On the global dimension of residue rings. Pac. J. Math. 32, 345-349 (1970)

8. Fossum, R.M., Griffith, P.A., Reiten, I.: Trivial extensions of abelian categories. Lecture Notes in Mathematics, vol. 456. Springer, Berlin (1975)

9. Happel, D.: Triangulated categories in the representation theory of finite-dimensional algebras. London Mathematical Society Lecture Note Series, vol. 119. Cambridge University Press, Cambridge (1988)

10. Happel, D., Reiten, I., Smalø, S.O.: Tilting in abelian categories and quasitilted algebras. Mem. Am. Math. Soc. 120(575) (1996)

11. Hughes, D., Waschbüsch, J.: Trivial extensions of tilted algebras. Proc. Lond. Math. Soc. (3) 46(2), 347-364 (1983)

12. Keller, B.: On the construction of triangle equivalences. In: Derived Equivalences for Group Rings, Lecture Notes in Math., vol. 1685, pp. 155-176. Springer, Berlin (1998) 
13. König, S.: Tilting complexes, perpendicular categories and recollements of derived module categories of rings. J. Pure Appl. Algebra 73(3), 211-232 (1991)

14. Ladkani, S.: On derived equivalences of categories of sheaves over finite posets. J. Pure Appl. Algebra 212(2), 435-451 (2008)

15. Mac Lane, S.: Categories for the working mathematician. Graduate Texts in Mathematics, vol. 5. Springer, New York (1998)

16. Michelena, S., Platzeck, M.I.: Hochschild cohomology of triangular matrix algebras. J. Algebra 233(2), 502-525 (2000)

17. Miyachi, J.I.: Recollement and tilting complexes. J. Pure Appl. Algebra 183(1-3), 245-273 (2003)

18. Palmér, I., Roos, J.E.: Explicit formulae for the global homological dimensions of trivial extensions of rings. J. Algebra 27, 380-413 (1973)

19. Rickard, J.: Morita theory for derived categories. J. Lond. Math. Soc. (2) 39(3), 436-456 (1989)

20. Tachikawa, H., Wakamatsu, T.: Applications of reflection functors for self-injective algebras. In: Representation Theory, I (Ottawa, Ont., 1984), Lecture Notes in Math., vol. 1177, pp. 308-327. Springer, Berlin (1986) 